\title{
A Case of Campylobacter Colitis Presenting as Pseudoappendicitis and Review of the Literature
}

\author{
Eric P. Zhou, Daniel J. Goldberg,, Eric E. Jung,, Annmarie Dunican,2 and Daithi S. Heffernan ${ }^{1,2}$
}

\begin{abstract}
Background: Right lower quadrant pain is evaluated commonly by the general surgeon. Appendicitis is a common presenting pathology, however, there are numerous other non-surgical pathologies that may present similarly.

Case Presentation: We present the case of a 37-year-old Caucasian male presenting with two days of right lower quadrant abdominal pain with elevated temperature, leukocytosis, diarrhea, and chills after preparing turkey for a Thanksgiving meal. On presentation, the patient was febrile and persistently tachycardic with a heart rate of 130 beats per minute. Imaging findings were consistent with ascending colitis with mesenteric adenitis. Stool studies were positive for Campylobacter. The patient was treated successfully non-operatively with antibiotics, fluid replacement, and bowel rest.

Conclusion: Patients who present with persistent right lower quadrant abdominal pain, leukocytosis, elevated temperature, nausea, vomiting, and diarrhea should be evaluated for acute appendicitis. However, multiple nonsurgical etiologies presenting as pseudoappendicitis must also be considered.
\end{abstract}

Keywords: appendicitis; Campylobacter; colitis; infectious; pseudoappendictis

\begin{abstract}
A PPENDICITIS is one of the most common pathologies encountered by the general surgeon [1]. The classic presentation involves a young patient presenting initially with vague peri-umbilical abdominal pain that had migrated to the right lower quadrant (RLQ). This may be associated with anorexia, elevated temperature, and nausea. The general surgeon will gravitate naturally to a surgical diagnosis in a patient who presents in such a manner. However, other entities such as inflammatory bowel disease [2], typhlitis, cecal diverticulitis, and infectious colitis may manifest in a similar manner. It is essential to remain cognizant of these other diagnoses. We describe a case of Campylobacter colitis presenting as a pseudoappendicitis and issue a cautionary note in the management of patients presenting with RLQ abdominal pain associated with diarrheal episodes.
\end{abstract}

\section{Case Presentation}

A 37-year old Caucasian male presented to the emergency department with a two-day history of diffuse abdominal discomfort and malaise, followed by two more days of severe focal RLQ abdominal pain. This pain was associated with subjective elevated temperatures, chills, and nausea. Intriguingly, the patient did endorse several episodes of loose stool. He had been in his usual state of health previously with no recent travel and no sick contacts. He had been the only household member preparing turkey for a Thanksgiving meal. His past medical history was significant for obstructive sleep apnea on continuous positive airway pressure (CPAP) therapy and gastroesophageal reflux disease. Past surgical history was non-contributory. Family history was positive for colorectal cancer for which the patient recently had undergone a high-risk screening colonoscopy without significant findings.

Physical examination revealed a diaphoretic male with an obese, non-distended abdomen with focal RLQ tenderness to palpation and no Rovsing or peritoneal signs. He was tachycardic on presentation with a heart rate of 130 beats per minute, normotensive, and a temperature of $38.6^{\circ} \mathrm{C}$. Laboratory studies revealed a leukocytosis of $14,200 \mathrm{~g} / \mathrm{dL}$ with left shift. All other laboratory work-up including lactate, lipase, and urinalysis were normal. A computed tomography (CT) scan with intravenous (IV) contrast was performed with

\footnotetext{
${ }^{1}$ Department of Surgery, Rhode Island Hospital, Providence, Rhode Island.

${ }^{2}$ Providence Veterans Affairs Medical Center, Warren Alpert Medical School of Brown University, Providence, Rhode Island.

(C) Eric P. Zhou et al. 2017; Published by Mary Ann Liebert, Inc. This Open Access article is distributed under the terms of the Creative Commons License (http://creativecommons.org/licenses/by/4.0), which permits unrestricted use, distribution, and reproduction in any medium, provided the original work is properly credited.
} 
findings of cecal and ascending colon wall thickening with prominent peri-cecal and right external iliac chain lymph nodes (Fig. 1) suggestive of ascending colitis with mesenteric adenitis. A normal-appearing appendix was visualized and no free fluid or air. Stool ova, parasites, cultures (Campylobacter, Salmonella, Shigella) and a Clostridium difficile toxin test were sent.

The patient was admitted and commenced on IV fluid resuscitation and empiric intravenous piperacillin-tazobactam administration. On hospital day two, he experienced multiple episodes of loose stools with fevers spiking to $39.4^{\circ} \mathrm{C}$, persistent RLQ abdominal pain requiring IV narcotics, and a persisting leukocytosis of $14,600 \mathrm{~g} / \mathrm{dL}$. On hospital day three, stool work-up returned as positive for Campylobacter antigen. Also, on hospital day three the patient began to show clinical signs of improvement with considerably less RLQ abdominal tenderness. This was associated with being afebrile and normalization of his white cell count. The patient was transitioned to oral levofloxacin. On hospital day four the patient was able to tolerate a normal diet. The patient was discharged on hospital day five with full toleration of diet and resolution of diarrhea, elevated temperature, leukocytosis, and abdominal pain.

\section{Discussion}

Appendicitis is caused by an initial obstruction of the appendiceal lumen. The sequelae of this inciting event lead to inflammation from bacterial growth, causing further venous congestion, arterial insufficiency, necrosis, and eventual perforation. Because of various anatomic variants of the appendix, diagnosis can often be a challenge. Classically, the inflamed appendix will come into contact with the peritoneum, causing the patient to have corresponding pain in the RLQ. Although patients with appendicitis may present with an episode or two of loose stool, it is not the predominant symptom. Indeed patients who present with RLQ abdominal pain and diarrhea need to have an alternative diagnosis considered. Pseudoappendictis is used to describe one of multiple pathologies, including a variety of infectious etiologies [3], that may mimic clinical symptoms of appendicitis without having the presence of an inflamed appendix. Severe diarrhea is defined as more than four fluid stools per day for more than three days. It is caused by a bacterial pathogen in $87 \%$ of cases [4]. Ultrasonography may be helpful in distinguishing bacterial ileocecitis from appendicitis. Ultrasound findings in patients with bacterial enteritis characteristically demonstrate enlarged lymph nodes and mural thickening of the terminal ileum and cecum [5]. The use of CT in the emergent work-up of patients with abdominal pain may also be helpful if the diagnosis of appendicitis is in doubt. Selective utilization of CT has been shown to decrease the incidence of negative appendectomy [6]. In our case report, the CT imaging showed cecal colitis with associated mesenteric adenitis, and most importantly a normalappearing appendix.

Because of the differing management of Campylobacter colitis versus appendicitis, it is imperative for the general surgeon to consider potential infectious differential diagnoses as well as be aware of the variation in required

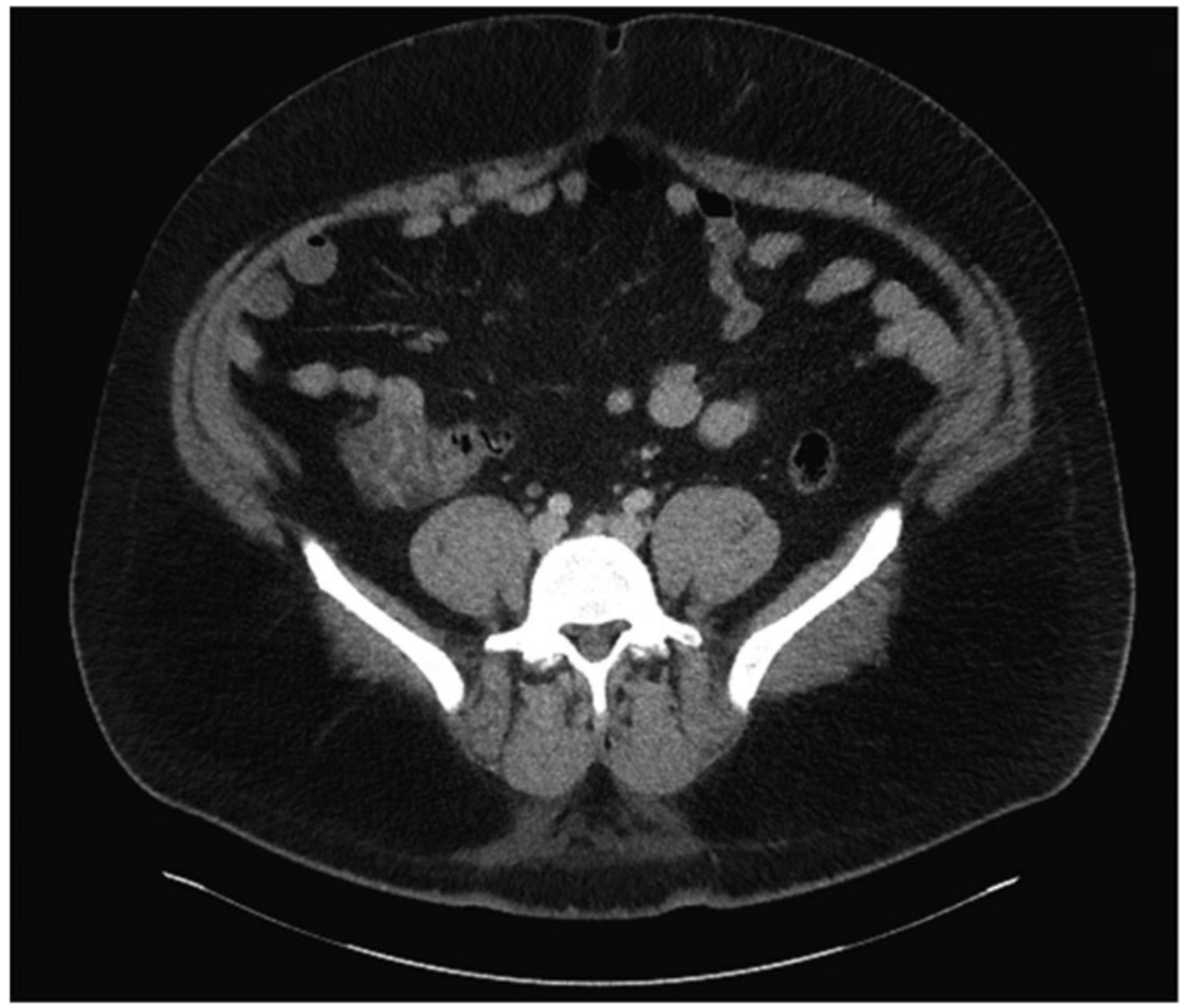

FIG. 1. Computed tomography scan demonstrating cecal colitis with mesenteric adenitis. 
antimicrobial management [7]. Most infections are selflimited and treatment is focused on adequate hydration and electrolyte repletion. Initiation of early antibiotics within the first three days of symptoms will reduce intestinal symptoms [8]. More severe symptoms such as high temperature, nonresolving symptoms, and bloody stools may require up to 14 $\mathrm{d}$ of antibiotic therapy. Macrolides, fluoroquinolones, aminoglycosides, and carbapenems have all shown to be effective against Campylobacter infection [9].

In the United States, Campylobacter is the most common cause of bacterial food-borne illness and is estimated to affect more than 2.4 million persons every year [9]. There is an estimated 76 million annual cases of food-borne illness in the United States. Humans become infected through consumption of Campylobacter-contaminated food, with poultry as a main reservoir of the disease. Unsafe food-handling practices have been linked to transmission of Campylobacter infections [10]. Non-specific clinical symptoms including abdominal pain, diarrhea, malaise, nausea, vomiting, and dizziness are reported. Because of the non-specific constellation of symptoms, Campylobacter infection can manifest itself as pseudoappendicitis, leading to the potential for an unnecessary appendectomy. We suggest recommend stool studies in patients presenting with acute abdominal pain, severe diarrhea, and non-diagnostic appendiceal imaging.

\section{Conclusion}

Patients who present with persistent RLQ abdominal pain, elevated temperature, nausea, and diarrhea should be evaluated for appendicitis. However, the general surgeon should also consider other non-surgical etiologies for RLQ abdominal pain that may manifest as pseudoappendicitis. We present a case of Campylobacter colitis as one etiology for RLQ abdominal pain. Clinically, the association of RLQ pain with diarrhea should lead surgeons to exercise caution prior to committing to operative intervention for RLQ pain.

\section{Author Disclosure Statement}

No competing financial interests exist.

\section{References}

1. Correa J, Jimeno J, Vallverdu H, et al. Correlation between intraoperative surgical diagnosis of complicated acute appendicitis and the pathology report: Clinicial implications. Surg Infect 2015;16:41-44.

2. Tierney J, Melvin W, May A, Bonatti H. Non-operative management of acute appendicitis in a patient with ulcerative colitis. Surg Infect Case Rep 2016;1:161-164.
3. Anthony C, Tessier J, Sanders J, et al. Streptococcal toxic shock syndrome presenting as an acute abdomen. Surg Infect Case Rep 2016;1:82-84.

4. Dryden M, Gabb R, SK W. Empirical treatment of severe acute community acquired gastroenteritis with ciprofloxacin. Clin Infect Dis 1996;22:1019-1025.

5. Puylaert J, Vermeijden R, van der Werf S, et al. Incidence and sonographic diagnosis of bacterial ileocaecitis masquerading as appendicitis. Lancet 1989;2:84-86.

6. DeArmond G, Dent D, Myers J, et al. Appendicitis: Selective use of abdominal $\mathrm{CT}$ reduces negative appendectomy rate. Surg Infect 2003;4:213-218.

7. Sartelli M, Catena F, Ansaloni L, et al. Duration of antimicrobial therapy in treating complicated intra-abdominal infections: A comprehensive review. Surg Infect 2016;17:9-12.

8. Ternhag A, Asikainen T, Giesecke J, Ekdahl K. A metaanalysis on the effects of antibiotic treatment on duration of symptoms caused by infection with Campylobacter species. Clin Infect Dis 2007;44:696-700.

9. Kirkpatrick B, Tribble D. Update on human Campylobacter jejuni infections. Curr Opin Gastroenterol 2011;27:1-7.

10. Redmond E, Griffith C. Consumer food handling in the home: A review of food safety studies. J Food Prot 2003; 66:130-161.

Address for correspondence: Dr. Daithi S. Heffernan Division of Surgical Research

Department of Surgery 205 Aldrich Building

Rhode Island Hospital 593 Eddy Street

Providence, RI 02903

E-mail: DHeffernan@brown.edu

$\begin{aligned} & \text { Abbreviations Used } \\ \mathrm{CPAP} & =\text { continuous positive airway pressure } \\ \mathrm{CT} & =\text { computed tomography } \\ \mathrm{IV} & =\text { intravenous } \\ \mathrm{RLQ} & =\text { right lower quadrant }\end{aligned}$

Cite this article as: Zhou EP, Goldberg DJ, Jung EE, Dunican A, Heffernan DS (2017) A case of Campylobacter colitis presenting as pseudoappendicitis and review of the literature. Surgical Infections Case Reports 2:1, 20-22, DOI: 10.1089/crsi.2017.0001 